\title{
Kriterien für einen guten Kinder- und Jugendfilm
}

\author{
von Anton Täubl
}

Für viele Erzieher, Pädagogen und Kulturpolitiker, aber auch für Bildungsmultiplikatoren im kirchlichen Dienst und für Pfarrer erhebt sich immer wieder die Frage nach Kriterien zur Beurteilung von Filmen, die für Kinder und Jugendliche geeignet sind; eine besondere Bedeutung erhält dieser Problemkreis für alle, die anfangen, ein Kinder- und Jugendfilmprogramm systematisch aufzubauen und nicht zuletzt für die Produzenten solcher Medien selbst.

Eine Auflistung einzelner Kriterien, die den Vorstellungen der Erwachsenen und in gleicher Weise den Bedürfnissen von Kindern und Jugendlichen standhalten soll, bereitet Schwierigkeiten: zum einen müßten solche Kriterien unter Berücksichtigung entwicklungspsychologischer Faktoren noch viel stärker als ich es hier tue auf die jeweiligen Altersgruppen hin ausdifferenziert werden; zum anderen bekommen die angeführten Kriterien einen jeweils anderen Stellenwert gemäß den Erziehungsvorstellungen und dem Bildungsbegriff, dem Wertsystem und der (religiösen) Weltanschauung, denen sich der verantwortliche Erzieher oder Bildungsreferent verpflichtet fühlt. Diese Problematik vorausgesetzt, möchte ich nachfolgend Kriterien darlegen, die als Diskussionsgrundlage zur weiteren Differenzierung und Objektivierung dienen können. So ein Kriterienkatalog verfolgt das Ziel, den jeweiligen Film für eine vor allem erzieherisch-pädagogische Bewertung faßbar zu machen, ohne die Subjektivität der Beurteilung ganz außer Acht zu lassen.

\section{Umschreibung des Begriffs „Film“ für Kinder und Jugendliche}

Die Bezeichnung „Kinder- und Jugendfilm“ soll hier weit gefaßt werden und sich nicht nur auf solche Angebote beschränken, die sich betont „kindertümlich“ beziehungsweise ,jugendgemäß" geben. Danach sind Kinder- und Jugendfilme Produktionen, die sich in erster Linie an Heranwachsende wenden und in der Auswahl von Themen wie in ihrer cineastischen Gestaltung auf diese Zielgruppe Rücksicht nehmen. Es sind Angebote, die ein weites Spektrum verschiedenartiger Inhalte und Formen enthalten und damit die Grundlage für ein differenziertes Film- und Wirklichkeitsbewußtsein schaffen, ohne sich dem Unterhaltungs- und Erlebnisbedürfnis der Kinder und Jugendlichen zu versagen. In solchen Filmen können Kinder und Jugendliche sich selbst mit ihren Zweifeln, Fragen, Wünschen und Sehnsüchten, sowie in ihren Lebensbedingungen und Umweltsituationen wiedererkennen und darüber mit anderen diskutieren. Unter diesem Blickwinkel ist jeder für Kinder und Jugendliche verständliche, für ihre Entwicklung förderliche und sie interessierende Film eine gute Produktion. ${ }^{1}$ Das bedeutet, daß eine gewaltsame Unterscheidung in Unterhaltung, Pädagogik und Kunst bei Kinder- und Jugendfilmen letztlich überflüssig ist, weil in den wirklich wertvollen Produktionen all diese Dimensionen enthalten sind. Das heißt aber auch, daß die landläufige Ansicht, ein Film mit oder über Kinder und Jugendliche sei automatisch auch schon ein „guter“ Film für dieses Publikum, keineswegs zutreffen muß. ${ }^{2}$

Anton Täubl ist Produktionsreferent am Institut für Film und Bild in Wissenschaft und Unterricht (FWU), Grünwald/München. 


\section{Kriterien, die sich auf die Gestaltung beziehen}

Es geht hierbei um die Frage, wie ein Film gemacht sein muß, wie er aussehen soll, damit er Kindern und Jugendlichen gefällt und seinen unterhaltsamen wie pädagogischen Wert trotzdem nicht verliert. In dem Zusammenhang scheinen mir folgende vier Gesichtspunkte von Bedeutung zu sein:

\subsection{Filmtechnische Aspekte}

Filme für Kinder und Jugendliche sollen mediengerecht gestaltet sein, das heißt, der filmtechnische Standard des Produkts muß zunächst einmal ein hohes Niveau aufweisen. Zu einer optimalen filmischen Gestaltung gehören: gute Bildqualität (Schärfe, Brillanz der Farben, richtige Helligkeitswerte), professioneller Schnitt (ohne Blitzer, der Thematik angepaßte Blenden, keine falsch angewandte Schnittechnik), akustisch einwandfreier und verständlicher Ton (Geräusche, Sprache, Musik, Mischung und so weiter); diese Forderung gilt auch für Kamera- und Regiearbeit (von der Aussage her gewählte Einstellungen, Schwenks oder Zooms; souveräne Führung des Kameramanns und der Rollenträger, gute Inszenierung) sowie für die Leistungen der Schauspieler (Ausdrucksfähigkeit, glaubwürdige Darstellung der Rolle). Von den Finanzen und vom Aufwand her können, dürfen Filme für Kinder und Jugendliche demnach keine „billigen“ Filme sein.

Da die Herstellung von Kinder- und Jugendfilmen sehr viel zusätzliche Arbeit erfordert und somit anderen Produktionsbedingungen unterliegt als herkömmliche Dreharbeiten (erwähnt seien beispielsweise nur einengende arbeitsrechtliche Bestimmungen für die Beschäftigung von Kindern, Betreuung der Kinder bei Aufnahmearbeiten, ständig neue Motivierung der Kinder- und Jugendstars), ist hier ein besonderes Verständnis und auch eine spezifische Hilfe dringend erforderlich: Staat und Gesellschaft - und damit auch die Kirchen - sie alle sind als Träger von Erziehung, Bildung und Kultur aufgerufen, den Kinder- und Jugendfilm aus dem Zwangssystem einer bloßen Profitmaximierung herauszulösen, was zum Beispiel in den skandinavischen und sozialistischen Ländern des Ostblocks bereits seit Jahren gelungen ist und zu einem beachtlichen Stand im Bereich der Kinder- und Jugendfilmkultur geführt hat. Auf lange Sicht dürfte das allerdings nur möglich sein durch eine großzügige Subventionierung der Produktions- und Vertriebsarbeit einerseits und durch die Förderung von Abspielstellen andererseits - auch außerhalb der kommerziell ausgerichteten Kinoketten, zum Beispiel in Form von kommunalen Kinos, Club-Kinos, Nachbarschafts-Kinos oder Pfarrkinos.

\subsection{Filmästhetische und filmdramaturgische Aspekte}

Jeder Film lebt von der gelungenen ästhetischen und dramaturgischen Verarbeitung der Problematik. Aufmerksamkeit und Betroffenheit des Publikums, Zu- oder Abneigung der Zuschauer gegenüber Handlungsträgern und Filmgeschehen werden erreicht durch die audiovisuell raffinierte Darstellung elementarer Empfindungen wie zum Beispiel freie Entfaltung, Sicherheit, Erfolg, Freude und Liebe, beziehungsweise Angst, Gefangenschaft/Unfreiheit, Niederlage, Trauer und Haß. Eine Idee muß sich entwickeln, der Inhalt muß Gestalt annehmen, die Filmstory muß in Bewegung geraten und anschaulich aufgelöst werden. Dabei wechseln sich Phasen der Spannung und Entspannung immer wieder miteinander ab. So kommt es in den meisten Filmen zu einem Konflikt: Hauptdarsteller und Gegenspieler stehen sich gegenüber; oft sind es aber auch Meinungen, Einstellungen, politische Programme oder auch (religiöse) Weltanschauungen, die These und Antithese bilden. An gut gestaltete Filme für Kinder und Jugendliche ergeben sich meines Erachtens unter anderem folgende cineastische Forderungen: ${ }^{4}$ 
a. Ohne daß der Kinder- und Jugendfilm „verpädagogisiert“ werden soll, müssen die Adressaten doch die Funktion und die Bedeutung der in Filmen gebrauchten Symbole erkennen: die Filme müssen sich sprachlich und gestalterisch auf einer Ebene bewegen, die eine Verständigung zwischen dem jungen Publikum und dem Medium möglich macht. Dies bedeutet kommunikationstheoretisch zunächst, daß der Film mikro- und makrostrukturell eindeutige Beziehungen aufweist, daß er seine „Geschichte“ gestalterisch und inhaltlich dem Alter der Heranwachsenden und ihren Verständnismöglichkeiten angemessen entwickelt. Die Dramaturgie des Films hat die entwicklungspsychologische Situation und das Erfahrungsfeld der Betroffenen zu beachten, das heißt, die Filmgeschichte sollte linear erzählt werden, keine Brüche haben, komplizierte Handlungsverschachtelungen vermeiden, nicht durch zu viele Zeitsprünge verwirren und die Sichtweise der Kinder und Jugendlichen in Rechnung stellen. Formal und inhaltlich sollten die Filme, um Verständigungsschwierigkeiten bei jüngeren Kindern zu vermeiden, deshalb auch vor allem gradlinig sein, Vor- und Rückblenden sowie Parallelhandlungen - soweit überhaupt nötig - klar erkennbar machen, ein mittleres Erzähltempo sowie eine einfache, aber keineswegs eine simple, unbedarfte Symbolik in Wort und Bild verwenden.

b. Eine reflexive Verarbeitung des Filmgeschehens durch Kinder und Jugendliche, die über das reine Verständnis des Wahrgenommenen hinausreicht und didaktisch sowie pädagogisch absolut erwünscht ist, ist nur dann zu erwarten, wenn der Vorgang der Perzeption und Rezeption von Aufmerksamkeit und Konzentration begleitet ist und genügend Zeit für die Verarbeitung der Eindrücke läßt. Filme, die andauernd nur spannend sind, in denen immer etwas los ist, in denen eine Actionszene der anderen folgt, wo ein Höhepunkt den anderen jagt, ohne daß sie dramaturgisch aus Handlung und Umwelt herauswachsen - solche Produktionen fesseln bloß die Aufmerksamkeit und sprechen mit ihr verbundene Gefühle an, während der Vorgang der Reflexion des Gehörten und Gesehenen und damit die Einstellung der Kinder zur Filmhandlung unberücksichtigt bleiben. Daraus ergibt sich zwangsläufig die erzieherische Forderung an Kinder- und Jugendfilme, daß sie das genaue Hinsehen und Wahrnehmen begünstigen sollen, daß sie auch Pausen vorsehen - und dies gerade nach wichtigen Abschnitten - damit die Heranwachsenden die Filmhandlung interpretativ auf sich beziehen können. Erst die Möglichkeit zur Distanz vom unmittelbaren Erlebnisdruck und zum Überdenken des Gehörten und Gesehenen schafft Voraussetzungen für die Entwicklung eines kritischen Bewußtseins gegenüber den Medien und ihren Botschaften.

c. Jeder Film sollte ein „Leitmotiv“, eine Identifikationsfigur haben, an der sich durchgängig die Handlungsdynamik aufbaut. Das kann eine einzelne Person sein, ebensoaber auch eine Gruppe oder ein Tier. Je stärker sich Kinder und Jugendliche mit einer Person, Situation oder Thematik identifizieren können, desto mehr werden Aufmerksamkeit und Interesse angesprochen. Dabei legt das Alter der Kinderstars auch die Identifikationsmöglichkeiten fest. Größte Chancen zur Identifikation bestehen dort, wo das Alter des Publikums mit dem der Darsteller übereinstimmt. Diese zentrale Figur sollte nicht stereotyp gezeichnet und in ihren Verhaltensweisen fixiert sein, sondern im Filmverlauf eine nachvollziehbare Entwick1ung durchmachen; der „Held/Star“ muß lernfähig sein und trotz der oft wundersamen Eigenschaften ähnlichen Bedingungen unterworfen bleiben wie die zuschauenden Kinder und Jugendlichen. Die Zahl der handelnden Figuren sollte bei kleineren Kindern zwei bis drei nicht übersteigen; für den optischen Bereich gilt, daß die Personen nicht in Detail- oder Großaufnahmen, zum Beispiel nur ein Finger oder Auge, sondern eher ganzheitlich gezeigt werden. Die Kamerabewegung sollte zwar flexibel sein, jedoch auf ausschließliche Dynamisierung durch ständigen Einstellungswechsel, Schwenks und Zooms verzichten. Das Verständnis erleichtert auch ein 
möglichst einheitlicher und überschaubarer Handlungsort, andernfalls würde beijüngeren Kindern nur Verwirrung hervorgerufen. Bei Dialogen ist es angebracht, die wörtliche Rede wiederzugeben, die den Eindruck des Unmittelbaren und Authentischen vermittelt und somit der Spontaneität wie dem impulsiven Situationserleben der Heranwachsenden entgegenkommt. Unbekannte Bildsymbole und vor allem zu viele Fremdwörter oder zu lange Sätze lassen einen Film für kleinere Kinder ungeeignet erscheinen. Für Kleinkinder ist es überhaupt günstig, wenn kein Kommentar oder Dialog vorhanden ist allenfalls eine narrativ gestaltete Erzählung -, sondern nur Musik und Geräusche, weil das nicht vom Bild ablenkt und die Verbalisierung der Filmstory fördert.

d. Filme für Kinder und Jugendliche sollten in der Machart eher behutsam vorgehen und das Publikum nicht durch pausenlose Action-Szenen, durch übertriebene idyllische und sentimentale Szenen, durch überzogen emotionale und sensationelle Aufmachung oder durch ihre Informationsfülle überfahren und überfordern. Wie die Erwachsenen, so werden auch Kinder und Jugendliche durch das übliche Fernseh- und Videoprogramm teilweise schon daran gewöhnt, an harten und ausgefallenen Sachen Geschmack zu finden. Deshalb haben zunächst Filme Erfolg, die viel Spannung und Kitzel, aufwendige Tricks und Action enthalten: Raserei, Schießen, Flucht, Verfolgung, Brutalitäten, exotische Milieus und phantastische Situationen. Ereignishaftigkeit und Handlungsfülle sind in Kinder- und Jugendfilmen notwendig, weil sie dem Erlebnis- und Tatendrang, weil sie der aktionistisch-motorischen Lebensphase vieler Kinder und Jugendlichen entsprechen. Es kommt aber einer Unterschätzung des jungen Publikums gleich, wenn man äußere Handlungen möglichst wechselhaft und turbulent zum Wesensmerkmal erklärt und dabei die Motivierung des Verhaltens, die inneren Beweggründe für das Auftreten der Darsteller als unwesentlich außer Acht läßt. Sind es komplexe Zusammenhänge, so sind sie nicht simplifiziert darzustellen, sondern vielmehr filmdramaturgisch so zu verarbeiten, daß ihre Mehrschichtigkeit auch für weniger lebenserfahrene kindliche Zuschauer erkennbar und verstehbar wird.

e. Bei Kinder- und Jugendfilmfestivals hat sich gezeigt, daß solche Produktionen am besten ankommen, die von einem guten Schuß Humor und Witz durchdrungen sind und die trotz der Widrigkeiten, die das Leben mit sich bringt von einem grundsätzlichen Lebensoptimismus und von unbefangener Lebensfreude getragen werden. Filme mit einer lebensbejahenden, warmherzigen Grundhaltung, die ein fröhliches Gemeinschaftsleben und intaktes Umweltverhalten darstellen, ohne zeitbedingte Konflikte und gesellschaftliche Probleme zu verharmlosen, bieten echte Lebenshilfe im Sinne von Zuspruch, Ermunterung und Orientierung; sie machen Mut zu eigener Erfahrung und übermitteln das dazugehörende Vertrauen. Manchmal kann man jedoch den Eindruck gewinnen, als hätten Filmemacher diese Überzeugungen inmitten des tristen Asphaltdschungels, der nervenden Plansolls und der drückenden Weltprobleme vergessen, so tiefsinnig-langweilig und lehrhaftmoralisierend, so bitter-ernst geht es in vielen ihrer Produktionen zu.

f. Die Laufzeit der Filme darf keine Fessel für den Inhalt sein. Auf 90 Minuten „gestreckte“ wie auf 30 Minuten „komprimierte“ Themen sollten vermieden werden! Für Kleinkinder wäre generell ein Angebot von kürzeren Filmen notwendig! Denn der erste, regelmäßige Umgang mit Filmen sollte eigentlich jenseits des Fernsehens beginnen: anhand von Einzelbildern, aber auch von kleinen gut überschaubaren Filmsequenzen, die man oft wiederholt, nachspielt oder bespricht und anhand von kurzen Geschichten, die einen Bezug zur gegenwärtigen oder künftigen Lebenssituation von Kindern haben. Eine Geschichte wiedergeben, Szenen im Detail schildern, in Rollen schlüpfen, Szenen nachspielen - das schult die Wahrnehmung, das Denken in Zusammenhängen und das 
Urteilen. Es fördert die Konzentration und sensibilisiert für die wahrgenommenen, bewegten Bilder. Das begünstigt sowohl den Umgang mit der Realität, die hinter den Filmen steht, wie auch die eigene Vorstellung und Phantasie. Erzieher und Eltern müssen bereits bei den jüngsten Fernsehzuschauern und Videofans darauf achten, da $B$ Bilder nicht die Sprache und die Kreativität verdrängen und damit die eigene gedankliche Arbeit und Entscheidungsfreude.

g. Je reifer, lebenserfahrener und verständiger die Heranwachsenden sind, um so eher können die auf (Klein-) Kinder abgestimmten filmischen Erzählweisen aufgebrochen werden: Mehrschichtigkeit in der Entfaltung der Thematik sowie Vor-und Rückblenden, das Spiel auf verschiedenen Handlungsebenen und der Wechsel von Zeitebenen, die offene Form mit ihrer Unabgeschlossenheit am Anfang und Ende der Film-Stories, Wechsel der Perspektiven und schnelle Schnitte, differenzierte soziale, politische und weltanschauliche Argumentation - all das erscheint zunehmend möglich und erweitert damit die cineastischen Gestaltungsmöglichkeiten.

\subsection{Entwicklungspsychologische Aspekte}

Ein wichtiges Kriterium für Kinder- und Jugendfilme besteht in ihrer Zuordnung zur entsprechenden Altersgruppe, was vor allem entwicklungspsychologisch und von Bildungsstand und Soziologie her auch schichtenspezifisch definiert werden muß. Obwohl die Grenzen fließend sind und jede Alterseinteilung, wie Zuweisung zu gesellschaftlichen Schichten auch problematisch bleibt, möchte ich auf folgende Gruppierungen aufmerksam machen:

a. Vorschulkinder: Das (Klein-) Kind gelangt vor allem handelnd zu einem Erfahrungswissen seiner Umwelt: nur was es mit allen Sinnen ständig erlebt und immer von Neuem ausprobiert, wird Bestandteil seiner Welt. Diese unmittelbare, direkte Begegnung mit der Umwelt ist durch kein Medium zu ersetzen. Das Leben der jüngsten Filmkonsumenten wird nach Jean Piaget dadurch gekennzeichnet, daß Kinder zwischen dem zweiten und vierten Lebensjahr in der Phase des „symbolisch-vorbegrifflichen“ Denkens leben, das dann vom vierten bis zum siebten Lebensjahr allmählich übergeht in die ,physiognomisch-anschauliche “ Welterfassung, die sich später zum „logisch-operativen“ Verhalten weiterentwickelt. Der junge Filmfan kann nur solche Eindrücke von der Kinoleinwand aufnehmen und sinnvollerweise mit der eigenen Lebenssituation verknüpfen, für die er bereits konkrete Vorlagen besitzt: seine unmittelbar erlebte Umwelt (Familie, Nachbarschaft, Kindergarten, Schule, Stadtteil; Freunde, Verwandte, Bekannte, Tiere und Pflanzen; Spielsachen und Spiele, Gegenstände des täglichen Bedarfs mit ihren Regeln und ihrem Funktionieren). Die meisten Erfahrungen werden erst dadurch zum Besitz des Kindes, daß sie in Eigentätigkeit umgesetzt werden - was medienpädagogische Konsequenzen für die Nacharbeit von Filmen nach sich zieht. Vom vierten Lebensjahr an beginnt das Kind ,als - ob - Spiele“ (Fiktionsspiele) mit anderen Personen gemeinsam zu spielen; solche Rollenspiele sind unentbehrlich für die kognitive, emotionale und soziale Entwicklung des Kindes. ${ }^{5}$

b. Bis acht Jahre: Das Kind hat seine Ich-Findung weitgehend abgeschlossen. Durch Identifikation und Internalisierung hat es relativ rigide Verhaltensformen und Wertstrukturen aufgebaut. Dieses kindliche Weltbild erweist sich da und dort, vor allem, wenn das Kind in Krisensituationen hineingerät, noch als brüchig. Seine Umwelt ist begrenzt auf Schule, Freunde, Elternhaus, nähere Umgebung und auch die Welt des Fernsehens, soweit sie dem Kind zugänglich ist. Größere gesellschaftliche Zusammenhänge werden nur punktuell und erst allmählich bewußt. 
c. Acht bis zwölf Jahre: Vereinheitlichung der Erfahrungswelt und der Erfahrungsmuster. Interessen verlagern sich von der eigenen Familie in den Freundeskreis; häufig tritt Cliquen- und Bandenbildung auf. Schulprobleme machen sich verstärkt bemerkbar. Einsetzende Pubertät führt zu Identifikationsschwierigkeiten mit der eigenen Rolle und Stellung. Widersprüchliches Verhalten zum anderen Geschlecht.

d. Zwölf bis 16 Jahre: Erfahrungsraum „peer group“. Allmähliches Abklingen der pubertären Unsicherheiten. Hinfindung zur geschlechtsspezifischen Identität. Beginn des Lebens als „soziales“ Wesen, das heißt, zunehmende Wahrnehmung der gesellschaftlichen Umwelt und ihrer Probleme, zum Beispiel im Zusammenhang mit der Berufswahl. Ausbruchs- und Fluchttendenzen aus bisherigem familiären und sozialen Umfeld. Versuch, Sehnsüchte und Lebensvorstellungen zu verwirklichen. Erste erotische Beziehungen durch Hinwendung zum anderen Geschlecht.

e. Über 16 Jahre: Verstärktes Hineinwachsen in Beruf, soziales Umfeld und Gesellschaft. Wachsende Selbständigkeit bei Übernahme vermehrter Verantwortung. Zunehmende Gewinnung einer politischen, sozialen, kulturellen und weltanschaulichen Position.

Bei Herstellung und Verwendung von Filmen für Kinder und Jugendliche sind entsprechende anthropologisch-psychologische Voraussetzungen und sozio-kulturelle Vorgegebenheiten zu berücksichtigen: Alter, Geschlecht, Reifegrad, Bildungsstand, Gesellschaftsschicht, Lebensmilieu. Es gilt immer wieder zu fragen: Wie kann durch den Film der Erfahrungsraum der Zielgruppe angesprochen werden? Welche Bedeutung hat der Film für die Heranwachsenden in ihrer Lebenswelt?

\subsection{Inhaltorientierte Aspekte (vgl. auch 3.)}

Unter didaktischen, pädagogischen und weltanschaulichen Gesichtspunkten betrachtet, dürfen Filme für Kinder und Jugendliche den Realitätsbezug und die Wahrheitsfrage nicht einfach als belanglos verdrängen oder gar ganz ausschalten.

a. Daraus ergibt sich zunächst einmal die Forderung nach sachlicher Übereinstimmung zwischen dem Film und der in ihm dargestellten Wirklichkeit (Stoff, Thema). Filme sollen die Inhalte eher der Sache angemessen und umfassend und nicht verkürzt oder verzerrt wiedergeben. Sie sollten Anregungen zur Auseinandersetzung mit den Problemen bieten und die Begegnung mit der Realität des Lebens nicht einseitig darstellen oder gar verhindern, etwa durch eine Anhäufung fiktiver Geschichten, durch die Aneinanderreihung vordergründiger Gags, durch die überzogene Ästhetisierung oder durch bewußte einseitige (Falsch-) Information.

Filme über biblische Themen sollten beispielsweise mehr sein als nur Anschauungsmittel oder Illustrationen, die sich in (pseudo-) historischen kulturgeschichtlichen oder geographischen Details erschöpfen. Hier gilt es die Unterscheidung von „Richtigkeit“ und innerer „Wahrheit" zu bedenken: Es gibt „richtige“ Aussagen, zum Beispiel in dem Film „Die Patriarchen" aus dem Genesis-Projekt (,new media bible"), wo bis ins Detail nach ägyptischen Vorlagen die Atmosphäre am Hofe des Pharao ausgestaltet wurde, die das Wesentliche jedoch kaum berühren, es eher verdunkeln als erhellen; „wahre“ Aussagen hingegen treffen nicht irgend etwas oder manches richtig, sondern sie legen das Wesentliche bloß, in diesem Fall den Kern der biblischen Botschaft, den Glauben des Abraham und Gottes Vorsehung. Ein Mehr an richtiger (Detail-) Information kann mit einem Weniger an substantieller Wahrheit durchaus Hand in Hand gehen. Will ein Film der Eigenart biblischer Texte und Erfahrungen gerecht werden, so wird er ihre kerygmatische Intention (Verkündungsabsicht) verstehen lernen und das in der Bibel verborgene 
Glaubensgut (Kerygma) zum Ausdruck bringen. Von einem „biblischen“ Film für Kinder und Jugendliche wird gewissermaßen eine audiovisuelle Exegese gefordert, die über Ohren und Augen zur Sinnmitte der Botschaft Jesu führt.

Auch die gesellschaftlichen Zustände müssen zutreffend wiedergegeben werden. Filme sollen dazu beitragen, daß sich Kinder und Jugendliche im Alltag besser zurechtfinden und die Ursachen der Ereignisse, von denen sie betroffen werden, allmählich begreifen. Das Postulat, auch in Kinder- und Jugendfilmen die Lebenswirklichkeit sachbezogen wiederzugeben, richtet sich gegen bedenkliche Absichten, die heranwachsenden $\mathrm{Zu}-$ schauer in eine mediale Scheinwirklichkeit zu entführen und sie von der Realität abzulenken, statt sie darüber aufzuklären.

b. Die Filmkunst schließt das Überzogene, Überdrehte, Einseitige, Ironische, Groteske, Phantastische und Fiktive nicht aus. Doch sollten Provokations-, Persiflagen-, Kabarett; Märchen-, Abenteuer- und Scienc-Fiction-Filme als solche erkennbar sein und ihre spezifische filmdramaturgische Wahrheitstiefe behalten. Die im Film gestaltete und wiedergegebene Welt muß jedenfalls Rückschlüsse auf die eigene Situation der Kinder und Jugendlichen erlauben, wobei es nicht erheblich ist, in welchem Milieu sich die Filmhandlung selbst abspielt: reale Umwelt oder exotische, beziehungsweise phantastische Umgebung. Die ästhetische Fiktion einer phantastischen Filmgeschichte hat durchaus ihre eigene Wahrheit! Die in der phantastischen Fiktion begründeten Titelfiguren vieler Filme sind zwar oft an den realen Raum und an die reale Zeit nicht gebunden, zeigen aber weiterhin doch menschliche Verhaltensweisen: sie können sich irren, Trauer und Freude zeigen, aus Erfahrung lernen; sie haben zwar wundersame Eigenschaften, diese aber bleiben ähnlichen Lebensbedingungen unterworfen, wie sie das Publikum kennt. Das gilt vor allem auch für Animationsfilme, in denen eher Sehnsüchte der Menschen als der konkrete Alltag, eher Utopien als triste Aktualität, eher das ideale Soll als das reale Ist, mehr das Sein an sich als das Haben zum Ausdruck kommen. Eine Trickfilmfigur überschreitet Raum und Zeit und begibt sich hinein in die Bereiche von Wünschen, der Phantasie und der Träume. Franz Kafkas Wort, „das wirkliche Realität immer unrealistisch ist“, trifft wie kaum ein anderes auf den Animationsfilm zu.

c. Gefährlich in dem Zusammenhang wird allerdings alles, was einer Idyllisierung, Sentimentalisierung und sensationellen Aufbauschung gleichkommt. Filme für Kinder und Jugendliche, deren Geschichten einseitig auf dem Hintergrund einer harmonistischen, gesichert-problemlosen bürgerlichen Welt beziehungsweise der ,geschlossenen“ Gesellschaft mit deren ideologisch ausgerichteten Moral- und sozialen Wertvorstellungen spielen, benötigen ein Kontrastangebot in Filmen, die bestehende Verhältnisse auch hinterfragen, Brüche im Leben des Einzelnen wie in der Gesellschaft aufzeigen und auch die Härten des Alltags erscheinen lassen. Der Rückzug in eine bloß private, familiäre, bürgerliche Atmosphäre unter Aussparung der sozialen Wirklichkeit muß immer wieder aufgebrochen und abgelöst werden durch Filme, die auch die ökonomischen, politischen, gesellschaftlichen und kulturellen Einbindungen und Beeinflussungen des Einzelnen und seiner Umwelt bewußt machen. Trivialitäten, Harmlosigkeiten, Simplifizierungen der Verhältnisse, Emotionalisierung der Zuschauer sowie penetrante Belehrung solcher Filme sind aber bedenklich, weil sie zu gefährlichen Entwicklungen im Bewußtsein der Kinder führen. Besonders die exzentrischen Erzählformen sind der Gefahr der Trivialisierung und der Verharmlosung ausgesetzt. Der idealistische Appell, die gesellschaftliche Realität zu verändern, verkommt allzu oft zur Einladung,dem Alltag den Rükken zu kehren; Phantasiewelten pervertieren zu bloßen Fluchträumen; Abenteuerhelden werden zu Kompensationsfiguren für die tatsächlichen Enttäuschungen, während ihre 
Gegenspieler als simple Sündenböcke für die Pannen im Leben und die Unzulänglichkeiten in der Gesellschaft herhalten müssen. Kinder, die etwa in Kriminal- und Westernfilmen, in Agenten; Kriegs- und Science-Fiction-Produktionen pausenlos mitansehen müssen, daß Beziehungen vor allem aus Konflikten zu bestehen scheinen und daß Probleme immer nur mit böser List und brutaler Gewalt gelöst werden, erhalten doch ein irreales Abbild von der Wirklichkeit. Viele Gestalten aus Fersehserien werden durch Schönheit, Jugend und Erfolg gekennzeichnet; diese Attribute spiegeln sich oft geradezu penetrant in der Wohnsituation wider, in den sozialen Kontakten, in Eß- und Trinkgewohnheiten, in Automarke und Kleidung. Not, Krankheit, Leiden, Altwerden, Tod, gesellschaftliche Diskriminierung und soziales Unrecht, aber auch weltanschauliche und religiöse Fragen bleiben weithin ausgeblendet, so als gäbe es sie nicht einmal.

d. Abzulehnen ist in Kinder- und Jugendfilmen eine rücksichtslose Indoktrination und eine die Wirklichkeit verfälschende Manipulation. Tatbestände und Fragen, die keine allgemeingültige und absolute Wertung zulassen, sollen auch von Filmen nicht abschlieBend, beziehungsweise als nicht mehr hinterfragbar beantwortet werden. Einseitig beeinflussende Filme - soweit sie überhaupt eingesetzt werden, etwa um zu provozieren und Emotionen zu wecken - müssen durch entsprechende Gegeninformationen, durch geeignete Kontrastmedien und -maßnahmen ergänzt werden, so daß den Betroffenen die Möglichkeit der kontroversen Auseinandersetzung und der freien Entscheidung eröffnet wird. Manipulativ gestaltete Produktionen, die absichtlich Informationen unterschlagen und Tatbestände verfälschen, um doktrinäre Zielsetzungen ökonomischer, politischer oder weltanschaulicher Art durchzusetzen, sind als solche zu entlarven. Auch Heranwachsende haben nämlich einen Anspruch auf Ehrlichkeit und Wahrheit darauf eben, daß man sie als Personen ernst nimmt.

\section{Kriterien, die sich auf die Inhalte beziehen}

Es handelt sich hier um eine im Detail zweifellos auch kontrovers zu erörternde Frage, nämlich welche Themen (Inhalte, Stoffe) für Kinder und Jugendliche geeignet sind und auf welche Weise sie in Filmen verarbeitet werden können. Im konkreten Fall wird die Beantwortung verschieden ausfallen, je nach Abhängigkeit des Urteilenden vom weltanschaulichen Umfeld - und damit vom sozio-ökonomischen Standard, von der gesellschaftspolitischen Lage und den pädagogisch-didaktischen Zielsetzungen, von den herrschenden Sitten und Gebräuchen, vom kulturellen Erbe der jeweiligen Gesellschaft.

a. Es ist zu wünschen, daß sich Kinder- und Jugendfilme von den Inhalten her grundsätzlich an den Interessen und Bedürfnissen, Vorlieben und Defiziten, Fragen und Problemen eben an den gegenwärtigen und künftigen Lebenssituationen der Kinder und Jugendlichen orientieren. In den Produktionen sollte das Fühlen und Denken der Heranwachsenden, ihre Einstellungen und Reaktionen, ihre Welt aufgegriffen und zum Ausdruck gebracht werden. Kinder und Jugendliche müssen sich und ihre Umwelt in den Filmen wiederfinden und ihren Sehnsüchten und Träumen nachhängen können.

b. Die natürliche, soziale und kulturelle Umwelt mit ihren Brüchen und Konflikten ist aus der Sicht, also im Blick auf die Auffassung von Kindern und Jugendlichen zu schildern. Solche Filme müßten Handlungsmuster anbieten - filmisch narrativ in anschaulichen Geschichten erzählt - die es in Auseinandersetzung damit Kindern und Jugendlichen erlauben, sich selbst ein Bild und eigene Gedanken zu machen, um ihre Lebensform zu finden und zu gestalten. Vor allem geht es um Beispiele von Rollenhandeln, die 
dem Erfahrungsraum von Kindern und Jugendlichen entspringen, beziehungsweise zugeordnet bleiben, mit denen sie sich identifizieren können, um diese Verhaltensweisen dann in „,innerer Auseinandersetzung“ oder bei ,praktischer Erprobung“ mit dem persönlichen Selbst in Beziehung zu setzen. Filme sollten Geschichten bringen, die Menschen (und Tiere) in konkreten Situationen zeigen und beschreiben, so daß Fragen, Probleme und Beziehungen zur Sprache kommen, die den Kindern und Jugendlichen auch den oft problematischen Alltag bewußt machen. Solche Produktionen müßten letzten Endes zur Erkenntnis des Gegebenen, des Alltäglichen, des Wirklichen sowohl im Sinne des Ist- als auch in dem des Sollzustandes hinführen und damit das Verlangen der Kinder und Jugendlichen nach Realitätserklärung und Daseinsbewältigung unterstützen.

c. Hinzu kommt die Forderung, daß Kinder und Jugendliche die Möglichkeit erhalten, anhand von Filmen aus der Geschichte zu lernen, beziehungsweise daß ihnen Geschichte erfahrbar gemacht wird, wozu beispielsweise auch Themenbereiche wie Aggression/ Gewalt/Krieg oder die jüngere deutsche Geschichte zählen. Das bedeutet aber nicht, daß sie im Film ständig mit den unbewältigten Problemen der Erwachsenen belangt werden müssen!

d. Eine Analyse der Inhalte von Filmen für Kinder und Jugendliche sowie eine Typologie der gängigsten Filmgenres die ja immer auch als Transportmittel für bestimmte Stoffe/ Themen in Erscheinung treten - zeigt folgendes Bild: ${ }^{6}$

- Filmthemen für Kinder und Jugendliche: Auf der Suche nach sich selbst - Arbeit, Beruf und Freizeit - Außenseiter, Randgruppen, Minderheiten - Behinderung und Krankheit - Dritte Welt - Drogen und Sucht - Einsamkeit und Isolation - Filmgeschichte Fluchtversuche/Aussteigerthematik - fremde Länder, fremde Völker, Reiseberichte Freund oder Feind, Freundschaft und Streit - Geschichte, Zeitgeschichte - Gewalt und Unterdrückung - große Komiker - Idole, Karriere - Kinder- und Jugendschicksale Kinder und Tiere - Konflikte im Alltag - Krieg und Faschismus (in Deutschland) - Leben im Heim - Lebensfragen, Sinnfragen - Lebensräume, Spielräume - Liebe, Ehe, Familie - Mädchen, Jungen - Märchen, Träume, Phantasien - modernes Leben, moderne Zeiten - Politik, Demokratie, Diktatur - Protest, Widerstand - Religion und Kirche - Schule und Erziehung - Selbstvertrauen, Ich-Findung - technischer Fortschritt und Zivilisation Sterben und Tod - Zeitkritik und Zukunftsfragen.

- Folgende Filmgenres sind bei Kinder- und Jugendfilmen besonders stark vertreten: Abenteuer - Bibliographien (Lebensschicksale) - Gangster- und Kriminalfilme Heimatfilme - Horrorfilme, Kino der Angst, Kriegs- und Antikriegsfilme - Komödien und Parodien - Kulturfilme - Literaturverfilmungen - Märchen, Sagen, Legenden Musikfilme - phantastische Filme, Science-Fiction-Filme - Politthriller - Tierfilme Western - Weltraum- und Zukunftsfilme.

\section{Zur Funktion von Filmen für Kinder und Jugendliche}

Welchen übergreifenden Zielsetzungen Filme funktional zu- und untergeordnet werden und welche Aufgaben sie im Leben von Kindern und Jugendlichen erfüllen sollen, hängt meines Erachtens von der kulturpolitischen Einstellung und damit vom jeweiligen Bildungsbegriff ebenso ab, wie vom Wertsystem und weltanschaulichen Standpunkt, denen sich der Filmverwender und die betroffenen Heranwachsenden selbst - bewußt oder unbewußt - verpflichtet fühlen. 


\subsection{Funktion der Unterhaltung, Zerstreuung, Freizeitgestaltung}

Filme im Kino sind zweifellos auch da, um Kinder und Jugendliche zu unterhalten und ihren Freizeitraum zu bereichern. Sie schaffen Vergnügen, dienen der Entspannung und Zerstreuung, amüsieren die Heranwachsenden. Beim unterhaltsamen Zuschauen bringen sie das Publikum zum Lachen und Weinen, zum Mitdenken und oft auch zum Verstehen der Verhältnisse. Als Alternative zum heimischen Fernseh-und Videokonsum holen Filme im Kino Kinder und Jugendliche aus der häuslichen Isolation heraus, um sie mit Gleichaltrigen zusammenzuführen. Durch dieses gemeinsame Filmsehen werden auch Gemeinschaftserlebnisse geschaffen, die für eine emotionale und soziale Entwicklung der Kinder und Jugendlichen nicht unterschätzt werden sollten. Filmerleben dieser Art schafft Raum, der nicht von Zweckdenken und Leistungsdruck geprägt wird, sondern als anregendes und erholsames Kontrastprogramm zu Schule, Ausbildung, Beruf und Elternhaus gilt. In dem Zusammenhang gewinnt eine Entwicklung an Bedeutung, die unter dem Schlagwort „Das andere Kino“ bekannt geworden ist. Schon seit Jahren zeigt sich, daß die kommerziellen Kinos nicht (mehr) in der Lage sind, Wünsche breiter Publikumsschichten zu befriedigen. Das hängt zum einen mit dem Kinosterben seit dem Aufkommen des Fernsehens Anfang der 50er Jahre zusammen, das bekanntlich dazu geführt hat, daß in vielen Dörfern und Städten die Lichtspieltheater ihre Tore schließen mußten; zum anderen liegt es häufig am miesen, einseitigen Programmangebot der noch vorhandenen Kinos - meist vordergründige Unterhaltung, häufig Gewalt und Pornographie - wodurch viele Erwachsene von einem Besuch abgehalten werden und Jugendliche als Publikum ohnehin nicht in Frage kommen. Der Werbeslogan „Mach Dir ein paar schöne Stunden, geh' ins Kino" erweist sich nur noch als bedingt zugkräftig. Seit einigen Jahren wird der Versuch unternommen, dem Film eine neue Wirkungsplattform bereitzustellen. Die neuen Bestrebungen wie beim kommunalen Kino, Club-Kino, Pfarr-Kino oder der Aktion guter Film wollen sich vom traditionellen Kinobetrieb nicht nur im Programmangebot unterscheiden; Filme sollen vielmehr auch in einer Umgebung vorgeführt werden, die das Gespräch mit dem Publikum ermöglicht und eine neue Verbindung zur Bildungsarbeit erschließt. Es kommt dabei vor allem darauf an, den Film in das Programm der Kinder- und Jugendarbeit sowie der Erwachsenenbildung zu integrieren. Die Durchführung solcher Veranstaltungen sollte sich von einer Kinovorstellung dadurch unterscheiden, daß beispielsweise durch Einführung oder durch Verteilung von schriftlichem Material ein Film erschlossen und die Möglichkeit der Weiterbearbeitung des Themas in Gesprächen, kreativen Gestaltungsarbeiten oder Rollenspielen angeboten wird. ${ }^{7}$

\subsection{Funktion der Information, Erziehung und Bildung}

Neben den Bedürfnissen nach Entspannung, Stillung des Erlebnishungers und Entschädigung für oft frustrierende Alltagserfahrungen im Sinne von Unterhaltungsbedarf, übernehmen Filme für Kinder und Jugendliche noch weitere wichtige Funktionen: Im Rahmen von Information, Erziehung und Bildung treten didaktische und volkspädagogische Aspekte wie Aufgaben der politisch-sozialen Orientierung in den Vordergrund. Zur Bildungsarbeit gehört es, die heranwachsende Generation zur Auseinandersetzung mit ökonomischen, politisch-sozialen und geistigen Strömungen der Zeit zu führen. Der Erfüllung dieser Aufgaben stellen sich heute insofern Hindernisse entgegen, als wir in einer Zeit der beschleunigten Veränderungen leben. Vieles von dem, war wir im Augenblick als bedeutend und bleibend empfinden, erweist sich manchmal schon in kurzer Zeit als unwichtig. Daraus ergibt sich, daß die gehäufte Vermittlung von Einzelinformationen immer weniger sinnvoll wird; eine auf das Kommende ausgerichtete Erziehung wird vielmehr bestrebt sein müssen, Heranwachsende zu befähigen, geistige Entwicklungen der 
Zeit und sich ankündigende Veränderungen an exemplarischen Phänomenen zu erkennen, sich mit ihnen auseinanderzusetzen und sich dann entsprechend zu verhalten. Statt viele Einzelfälle zu erörtern, werden prototypische Situationen gründlich reflektiert. In dem Zusammenhang wird die Arbeit mit Filmen bedeutsam, wenn sie Kindern und Jugendlichen dazu verhilft, Verständnis für Gegenwart und Zukunft an exemplarischmodellhaften Fällen zu gewinnen oder wenn sie Fragen auslösen, die für das Selbstverständnis des Glaubens in unserer Zeit grundsätzliche Bedeutung haben. Verdeutlichen läßt sich das beispielsweise an dem Legetrickfilm „Samariter 66“: Ein Ertrinkender winkt mit einem Bündel Banknoten einem Spaziergänger auf der Brücke; dieser springt ins Wasser, um das Geld zu holen! Beim Filmgespräch weisen die Zuschauer immer wieder auí einen Aspekt hin: Die Industrienationen beuten aus Egoismus, Eigennutz, Habgier, Gleichgültigkeit oder Skrupellosigkeit die Länder der Dritten Welt aus und lassen dabei die Menschen verkommen. Die Problematik des Nord-Süd-Dialogs wird schlagartig deutlich. Zu ähnlichen Ergebnissen führen die Kurzfilme „Hunger“ und „Auf der Kippe“.

Bei der zunehmenden Menge und unterschiedlichen Art von Eindrücken und Nachrichten ist der Heranwachsende allerdings nicht mehr in der Lage, die Informationen zu verarbeiten und zu ordnen. Die Fülle von Zahlen, Fakten und Meinungen oft gegensätzlicher Art orientieren nicht mehr, sondern desorientieren eher. Dadurch wird das unkritische Konsumverhalten weiter begünstigt. Auch kann der Nachrichtenansturm zu einer Abstumpfung gegenüber den vermittelten Geschehnissen führen: pausenlose Berichterstattung über Gewalttaten, Verbrechen, Katastrophen, Kriegsgreuel und Korruption rüttelt manche Zeitgenossen kaum mehr wach. Der Einzelne, der nicht gelernt hat die aus verschiedenen Quellen auf ihn zukommenden Informationen und Stellungnahmen zu vergleichen und abzuwägen, gerät damit in Gefahr, Opfer der Manipulation zu werden: statt eines wirklichen Wissens von Zuständen, tritt häufig ein verzerrtes und unvollständiges Bild der Wirklichkeit in den Vordergrund. So stehen wir heute vor dem schwierigen Problem, auf der einen Seite dafür zu sorgen, daß Kinder und Jugendliche nicht zu wenig Entwicklungsreize erhalten; und auf der anderen Seite, daß sie nicht durch eine Überfülle von Eindrücken überfordert werden. Hier zeigt sich eine neue Aufgabe von Elternhaus, Schule und außerschulischer Bildungsarbeit: nicht nur ständig Neues vermitteln, sondern die Fülle der Eindrücke bewältigen helfen; es gilt den Heranwachsenden beizustehen, damit sie sich mit all dem Neuen auseinandersetzen und es in ihr Weltbild integrieren. Durch gezielte medienpädagogische Maßnahmen kann diese kritische Haltung gegenüber den Medien gefördert werden.

\subsection{Förderung gesellschaftlicher Kommunikation und von Bürger-Initiativen}

$\mathrm{Zu}$ den wichtigsten Bedürfnissen der Heranwachsenden gehört es, mit Ihresgleichen in Kontakt zu treten, ihre Erlebnisse kundzugeben und eigene Vorstellungen zu formulieren. Der Drang zur Mitteilung von Gehörtem und Gesehenem, über Gefühle und Erfahrungen zu sprechen ist bei Kindern und Jugendlichen sehr groß. „Kommunikation“ hat einen hohen Stellenwert in der Filmarbeit. Sowohl praxisorientierte Filmpädagogik wie Medienforschung zeigen, daß bewegte Bilder eine stark aktivierende Kraft haben. Totalsprache, dramaturgische Gestaltung und der Vorgang der Identifikation sprechen das Publikum ganzheitlich an und erfassen dadurch nicht nur die rational-kognitive Ebene, sondern auch unterbewußte Schichten, den voluntativen und emotionalen Bereich. Zur Aufarbeitung von Filmen sollte daher nicht nur das Filmgespräch gehören, sondern alle weiteren Formen des kommunikativen Ausdrucks wie des entdeckenden und tätigen Lernens: zeichnerisches und malerisches Tun, Gestalten mit Knetmasse, Rollenspiele bis hin zu eigenen Filmproduktionen (aktive Medienarbeit), welche die Trennung von Medientheorie und Medienpraxis, von Wissen und Handeln aufheben. Gerade 
Letzteres, die Erweiterung der „privaten“ Kommunikation durch Vervielfältigung und Projektion eigener Filme führt zu einer „Veröffentlichung" der dargestellten Themen über die jeweilige Gruppe hinaus. Aktive Filmarbeit, vor allem die Selbstherstellung von Produktionen mit Kindern und Jugendlichen, erhöht und objektiviert die Bedeutung der privaten Aussagen, zwingt die Beteiligten aber zugleich zu genauer, fehlerfreier Ausführung, insbesondere, wenn eine Antwort erwartet wird (feed back, Rückkoppelung, Filmkorrespondenz). In dem Zusammenhang eröffnet sich die Möglichkeit, Filme auch als Mittel sozialer Bewußtseinsbildung wie politscher Arbeit zu verwenden, um Informationen zu verbreiten und Aktionen zu fördern, die sonst zu kurz kommen oder ganz unterbleiben würden. Damit läßt sich basisnahe Kommunikation und lokale Öffentlichkeit im kommunalen wie im kirchlichen Gemeindebereich herstellen. Der gesellschaftskritische und gesellschaftsverändernde Impuls vieler Filme ist ja unbestreitbar. Der gezielte Einsatz von Filmen schafft Problembewußtsein und vermittelt humanisierende Anstöße, die einer Verengung des Frage- und Denkhorizontes entgegenwirken. Der dokumentarische Kurzfilm „Ende des Dialogs“ gibt eine standortgebundene Darstellung der Rassendiskriminierung in Südafrika aus der Sicht unterdrückter Schwarzer wieder. Er konfrontiert die Zuschauer mit einer Gesellschaft, die Menschen in hochwertige und minderwertige einteilt und diese Verletzung der Menschenrechte aufgrund ideologischer Überzeugungen für gerechtfertigt hält. Der Film weist auf daraus resultierende Mißstände und Ungerechtigkeiten hin und fordert die "freie" Welt zur Stellungnahme heraus. Gerade „provokatorisch“ und „agitatorisch“ aufgebaute AV-Medien begünstigen emotional geladene Reaktionen und spontane Stellungnahmen der Anerkennung oder Ablehnung; sie bedürfen allerdings einer besonders sorgfältigen Aufarbeitung.

\section{Anmerkungen}

1 Vgl. dazu Hans Strobel: Der Kinderfilm - Kino für alle. In: Aufbruch zum neuen deutschen Kinderfilm, hg. von Wolfgang Schneider. Eulenhof-Verlag: Hardebek 1982, $245 \mathrm{ff}$.

2 Vgl. dazu auch Michael Hahn: Kinder- und Jugendfilmzentrum in der Bundesrepublik Deutschland (KJF). Projekt: Kinderfilmarbeit. KJF, Remscheid 1978, 19 f. Nur als Manuskript vorhanden.

3 Vgl. dazu Theda Kluth/Ulrike Fligers: Dem Kinderfilm eine Chance. Kinder- und Jugendfilmzentrum in der Bundesrepublik Deutschland, Remscheid 1982, 51 - 115.

4 Vgl. dazu Klaus Keller: Kinder und Film. Geschichte, Erforschung und Perspektiven des Kinderfilms, BAG Bundesarbeitsgemeinschaft der Jugendfilmclubs e. V.. Arbeitsgemeinschaft für Jugendfilmarbeit und Medienerziehung. Aachen 1978, $171 \mathrm{ff}$.

5 Vgl. Jean Piaget: Das Erwachen der Intelligenz beim Kind. Stuttgart: Klett-Verlag, 1969.

6 Dieser Zusammenfassung liegen Untersuchungen der Filmkataloge von atlas-film, des Kinderund Jugendfilmzentrums in der Bundesrepublik Deutschland, des Instituts für Film und Bild in Wissenschaft und Unterricht (FWU), des Katholischen Filmwerks GmbH sowie die Kurz- und Spielfilmliste des Gemeinschaftswerks der Evangelischen Publizistik e. V. zugrunde.

7 Vgl. dazu Hans May/Anton Täubl: Praxis AV-Medien. Anleitungen für Religionsunterricht und Kirchliche Bildungsarbeit. München: Kösel 1981, 46 ff. 


\section{SUMMARY}

In this contribution criteria are developed for a good childrens and youth film. The considerations are based on the conviction that such films have an entertaining, informative and communicative role. Content and form are to be distinguished. According to the author it is also essential that youth films do not exaggerate indoctrinal or informative tendencies. One has to respect psychological developments of young people. Especially short films should reflect the environment of the recipients, they should help towards a critical approach and new initiatives. The capability to digest the theme within a group with others seems to be especially important. In education and formation work, youth and childrens films have a special role to play.

\section{RÉSUMÉ}

L'auteur développe des critères à appliquer à un bon film pour enfants et jeunes, partant du fait qu'un tel film a des fonctions communicatives, informatives et divertissantes. Il distingue entre les critères du contenu et de la présentation. D'après Täubl, il est essentiel que des films de jeunesse ne se présentent ni avec des tendances indoctrinaires ni avec des tendances informatives exagerées. Mais il faut respecter les aspects psychologiques de développement des jeunes. Les films, notamment les courts métrages, utilisés dans la formation et le travail communautaire, devraient reflecter l'environment du public, stimuler la critique et des nouvelles formes d'action. Ce genre de film est important pour le traitement de groupe de la problématique du film. Dans le secteur de l'éducation, les films d'enfants et de jeunes ont un rôle important.

\section{RESUMEN}

En este articulo se dan a conocer algunos criterios acerca de los buenos films para ninos y jóvenes. Estas consideraciones se basan en la convicción que tales films deben cumplir un rol de entretención, de información y de comunicación. Se deben diferenciar los contenidos y la forma. Según el autor, es también esencial que no se exageren las tendencias informativas o de adoctrinamiento. Es necesario respetar el desarrollo psicológico de la juventud. En especial los films cortos pueden reflejar de mejor manera el medio ambiente de los expectadores y esto puede dar lugar a una aproximación crîtica y a nuevas iniciativas. La capacidad para asimilar el tema con otros al interior del grupo sería un aspecto muy importante. En el trabajo de educación y de formación, los films parajóvenes y ninos tienen un rol especial que cumplir. 